\title{
CONVERGENCE OF WEIGHTED AVERAGES OF RELAXED PROJECTIONS
}

\author{
RYSZARD SZWARC
}

\begin{abstract}
The convergence of the algorithm for solving convex feasibility problem is studied by the method of sequential averaged and relaxed projections. Some results of H. H. Bauschke and J. M. Borwein are generalized by introducing new methods. Examples illustrating these generalizations are given.
\end{abstract}

\section{Introduction AND PRELIMINARIES}

Many problems in applied mathematics deal with finding a point in the intersection of a family of convex sets in Euclidean or Hilbert space. The solution can be achieved in algorithmic way as a limit of composition of projection onto these convex sets. Due to its importance the problem has been studied heavily for many years. We refer to [1] where the reader can find a vast account of theorems and literature related to the problem, as well as a general approach which captures the earlier methods and results. In the present work we will generalize, in case of finite dimension, one of the main theorem of [1], concerning the convergence of algorithm. Firstly we will improve the estimate of concerning the average of relaxed projections, secondly we will admit repetitive control, and lastly we will make use of perturbation theorem, which allows to ignore projections with small weight coefficients. The first generalization is valid also in infinite dimensional inner product space. All these was possible by applying new techniques and new proof as well.

For a closed convex set $C \subset \mathbb{R}^{d}$ let $P_{C}$ denote the projection onto $C$. For $x \in \mathbb{R}^{d}$ the symbol $d(x, C)$ will denote the distance from $x$ to $C$. Assume we are dealing with a fixed finite family of closed convex sets $C_{1}, C_{2}, \ldots, C_{N}$. For a sequence of relaxation parameters $\alpha_{1}, \ldots, \alpha_{N}$

2000 Mathematics Subject Classification. Primary 49M45.

Key words and phrases. convex set, weighted projections, relaxation.

Supported by European Commission Marie Curie Host Fellowship for the Transfer of Knowledge "Harmonic Analysis, Nonlinear Analysis and Probability" MTKDCT-2004-013389 and by MNiSW Grant N201 054 32/4285. 
such that $0 \leq \alpha_{i} \leq 2$ and numbers $\lambda_{1}, \ldots, \lambda_{N}$ such that $0 \leq \lambda_{i} \leq 1$, $\sum_{i=1}^{N} \lambda_{i}=1$ we will consider the weighted averages

$$
\sum_{j=1}^{N} \lambda_{j}\left\{\left(1-\alpha_{j}\right) I+\alpha_{j} P_{C_{j}}\right\}=I-\sum_{j=1}^{N} \lambda_{j} \alpha_{j}\left(I-P_{C_{j}}\right) .
$$

It is well known that every operator $P_{C_{j}}$ is firmly nonexpansive (see [1, Facts 1.5]), thus these weighted averages are nonexpansive. Since the expressions depend only on the products $\lambda_{i} \alpha_{i}$ we will introduce the set

$$
\mathcal{B}=\left\{\beta=\left(\beta_{1}, \ldots, \beta_{N}\right) \mid \beta_{i} \geq 0, \sum_{i=1}^{N} \beta_{i} \leq 2\right\}
$$

and define for $\beta \in \mathcal{B}$

$$
Q_{\beta}=I-\sum_{j=1}^{N} \beta_{j}\left(I-P_{C_{j}}\right) .
$$

Remark. Observe that for any $\beta \in \mathcal{B}$ there exist relaxation parameters $\alpha_{1}, \ldots, \alpha_{N}$ and average parameters $\lambda_{1}, \ldots, \lambda_{N}$ such that $\beta_{i}=$ $\lambda_{i} \alpha_{i}$. Indeed, if $\sum_{k=1}^{N} \beta_{k}>0$ we may set $\alpha_{i}=\sum_{k=1}^{N} \beta_{k}$ and $\lambda_{i}=$ $\beta_{i}\left(\sum_{k=1}^{N} \beta_{k}\right)^{-1}$. On the other hand if $\beta_{i}=0$ for any $i$ we take $\alpha_{i}=0$ for any $i$ and $\lambda_{i}=1 / N$. Therefore every operator $Q_{\beta}$ is nonexpansive. Remark. All the results in this work remain valid if we replace $P_{C_{i}}$ with a firmly nonexpansive mappings $T_{i}$ such that $T_{i}(c)=c$ for $c \in C_{i}$ (see [1, p. 370]).

\section{Auxiliary Results}

Proposition 1. For any $x \in \mathbb{R}^{d}$ and any $c \in C_{1} \cap C_{2} \cap \ldots \cap C_{N}$ we have

$$
\left\|Q_{\beta}(x)-c\right\|^{2} \leq\|x-c\|^{2}-\sum_{j=1}^{N}\left(2-\frac{\beta_{j}}{\kappa_{j}}\right) \beta_{j}\left\|x-P_{C_{j}}(x)\right\|^{2},
$$

where $\kappa_{1}, \kappa_{2}, \ldots, \kappa_{N}$ are any nonnegative numbers such that $\sum_{j=1}^{N} \kappa_{j}=1$.

Remark. We set $\beta_{j} / \kappa_{j}=0$ whenever $\beta_{j}=0$. If $\beta_{j}>0$ and $\kappa_{j}=0$ we set $\beta_{j} / \kappa_{j}=+\infty$.

Proof. With no loss of generality we may assume that $c=0$. Then by the convexity of each set $C_{j}$ we have

$$
\left\langle P_{C_{j}}(x), x-P_{C_{j}}(x)\right\rangle \geq 0 .
$$


Hence

$$
\begin{aligned}
& \left\|Q_{\beta} x\right\|^{2}=\left\|x-\sum_{j=1}^{N} \beta_{j}\left(x-P_{C_{j}}(x)\right)\right\|^{2} \\
& =\|x\|^{2}+\left\|\sum_{j=1}^{N} \beta_{j}\left(x-P_{C_{j}}(x)\right)\right\|^{2}-2 \sum_{j=1}^{N} \beta_{j}\left\langle x, x-P_{C_{j}}(x)\right\rangle \\
& =\|x\|^{2}+\left\|\sum_{j=1}^{N} \beta_{j}\left(x-P_{C_{j}}(x)\right)\right\|^{2}-2 \sum_{j=1}^{N} \beta_{j}\left\|x-P_{C_{j}}(x)\right\|^{2} \\
& -2 \sum_{j=1}^{N} \beta_{j}\left\langle P_{C_{j}}(x), x-P_{C_{j}}(x)\right\rangle \\
& \leq\|x\|^{2}+\left\|\sum_{j=1}^{N} \beta_{j}\left(x-P_{C_{j}}(x)\right)\right\|^{2}-2 \sum_{j=1}^{N} \beta_{j}\left\|x-P_{C_{j}}(x)\right\|^{2}
\end{aligned}
$$

Let $\kappa_{1}, \kappa_{2}, \ldots, \kappa_{N}$ satisfy the assumptions. Then by the convexity of the function $x \mapsto\|x\|^{2}$ we obtain

$$
\begin{array}{r}
\left\|\sum_{j=1}^{N} \beta_{j}\left(x-P_{C_{j}}(x)\right)\right\|^{2}=\left\|\sum_{j=1}^{N} \kappa_{j} \kappa_{j}^{-1} \beta_{j}\left(x-P_{C_{j}}(x)\right)\right\|^{2} \\
\leq \sum_{j=1}^{N} \frac{\beta_{j}^{2}}{\kappa_{j}}\left\|x-P_{C_{j}}(x)\right\|^{2} .
\end{array}
$$

Combining (1) and (2) concludes the proof.

Remark. Setting

implies

$$
\kappa_{j}=\frac{\beta_{j}}{\sum_{k=1}^{N} \beta_{j}}
$$

$$
\left\|Q_{\beta}(x)-c\right\|^{2} \leq\|x-c\|^{2}-\left(2-\sum_{k=1}^{N} \beta_{k}\right) \sum_{j=1}^{N} \beta_{j}\left\|x-P_{C_{j}}(x)\right\|^{2}
$$

the inequality obtained in [1, Lemma 3.2(ii)].

Theorem 1. Given a family of convex sets $C_{1}, \ldots, C_{N}$ with nonempty intersection $C$. Let $\beta \in \mathcal{B}$ and $I \subset\{1,2, \ldots, N\}$. Then for any $x \in \mathbb{R}^{d}$ and any $c \in C$ we have

$$
\left\|Q_{\beta}(x)-c\right\|^{2} \leq\|x-c\|^{2}-\min _{i \in I} \nu_{i} \max _{i \in I} d^{2}\left(x, C_{i}\right),
$$


where

$$
\nu_{i}=\frac{2 \beta_{i}\left(2-\sum_{k=1}^{N} \beta_{k}\right)}{\beta_{i}+2-\sum_{k=1}^{N} \beta_{k}} .
$$

In particular the inequality holds when $I$ is the set of active indices, i.e.

$$
I=\left\{i \mid 1 \leq i \leq N, \beta_{i}>0\right\}
$$

Proof. Fix $i \in I$ and set

$$
\kappa_{j}= \begin{cases}\frac{1}{2} \beta_{j} & j \neq i, \\ 1-\frac{1}{2} \sum_{j \neq i} \beta_{j} & j=i .\end{cases}
$$

On substituting theses values into the inequality of Proposition 1 we obtain

$$
\left\|Q_{\beta}(x)-c\right\|^{2} \leq\|x-c\|^{2}-\nu_{i} d^{2}\left(x, C_{i}\right) \leq\|x-c\|^{2}-\min _{k \in I} \nu_{k} d^{2}\left(x, C_{i}\right) .
$$

Now maximizing with respect to $i \in I$ gives the conclusion.

\section{MAIN RESULT}

Theorem 2. Fix a family of convex sets $C_{1}, \ldots, C_{N}$ with nonempty intersection $C$. Given a sequence $\beta^{(n)} \in \mathcal{B}$. Let $I^{(n)}$ denote the set of active indices for $\beta^{(n)}$. Assume that every index $i \in\{1,2, \ldots, N\}$ occurs in $I^{(n)}$ for infinitely many $n$. Let $n_{k}$ be positive integers such that $n_{k-1}<n_{k}$ and

$$
\{1,2, \ldots, N\} \subset I^{\left(n_{k-1}\right)} \cup I^{\left(n_{k-1}+1\right)} \cup \ldots \cup I^{\left(n_{k}-1\right)},
$$

i.e. every index occurs at least once for $n$ such that $n_{k-1} \leq n<n_{k}$. For

let

$$
\nu_{i}^{(n)}=\frac{2 \beta_{i}^{(n)}\left(2-\sum_{k=1}^{N} \beta_{k}^{(n)}\right)}{\beta_{i}^{(n)}+2-\sum_{k=1}^{N} \beta_{k}^{(n)}} .
$$

$$
\nu^{(k)}=\min \left\{\nu_{i}^{(n)} \mid n_{k-1}<n \leq n_{k}, i \in I^{(n)}\right\} .
$$

Assume that

$$
\sum_{k=1}^{\infty} \nu^{(k)}=+\infty
$$

Then for any $x^{(0)} \in \mathbb{R}^{d}$ the sequence $x^{(n)}$ defined as

$$
x^{(n)}=Q_{\beta^{(n)}}\left(x^{(n-1)}\right), \quad n \geq 1
$$

is convergent to a point in $C$. 
Remark. This result generalizes [1, Thm 3.20(ii)] (see also [1, Cor. 3.25]) in two essential aspects. First of all it allows repetitve control while [1, Thm 3.20] could afford only intermittent control, i.e. when the sequence $n_{k}$ is of the form $n_{k}=k p$. Secondly the coefficients $\nu_{i}^{(n)}$ are smaller than $\mu_{i}^{(n)}$, introduces in [1] as

$$
\mu_{i}^{(n)}=2 \beta_{i}^{(n)}\left(2-\sum_{k=1}^{N} \beta_{k}^{(n)}\right) .
$$

For example let $N=2$ and

$$
\beta_{1}^{(n)}=\frac{1}{n}, \beta_{2}^{(n)}=2-\frac{2}{n} .
$$

The algorithm is then 1 -intermittent, hence we can take $n_{k}=k$ and $I_{k}=\{1,2\}$. Thus

$$
\sum_{k=1}^{\infty} \min \left\{\mu_{1}^{(k)}, \mu_{2}^{(k)}\right\}<+\infty, \quad \sum_{k=1}^{\infty} \min \left\{\nu_{1}^{(k)}, \nu_{2}^{(k)}\right\}=+\infty .
$$

Therefore Theorem 3.20 of [1] does not apply while our Theorem 2 does.

Proof. With no loss of generality we may assume that $0 \in C$. Fix $u^{(0)} \in \mathbb{R}^{d}$ and let $u^{(n)}=Q_{\beta^{(n)}}\left(u^{(n-1)}\right)$ for $n \geq 1$. By Theorem 1 we get

$$
\left\|u^{(n)}\right\|^{2} \leq\left\|u^{(n-1)}\right\|^{2}-\min _{i \in I^{(n)}} \nu_{i}^{(n)} \max _{i \in I^{(n)}} d^{2}\left(u^{(n-1)}, C_{i}\right)
$$

Iterating (6) leads to

$$
\begin{aligned}
& \left\|u^{(n)}\right\|^{2} \leq\left\|u^{(0)}\right\|^{2}-\sum_{m=1}^{n} \min _{i \in I^{(m)}} \nu_{i}^{(m)} \max _{i \in I^{(m)}} d^{2}\left(u^{(m-1)}, C_{i}\right) \\
& \leq\left\|u^{(0)}\right\|^{2}-\min _{\substack{0<m \leq n \\
i \in I^{(m)}}} \nu_{i}^{(m)} \max _{\substack{1 \leq m \leq n \\
i \in I^{(m)}}} d^{2}\left(u^{(m-1)}, C_{i}\right)
\end{aligned}
$$

Lemma 1. Given a family of convex sets $C_{1}, \ldots, C_{N}$ with nonempty intersection $C$. Given a sequence $\beta^{(n)} \in \mathcal{B}$. Let $I^{(n)}$ denote the set of active indices for $\beta^{(n)}$. Assume that every index $i \in\{1,2, \ldots, N\}$ occurs in $I^{(n)}$ for at least one $n$. Then for any positive number $R$ there exists a nondecreasing and positive function $\eta_{R}:(0,+\infty) \rightarrow(0,+\infty)$ such that

$$
\max _{\substack{n \geq 1 \\ i \in I^{(n)}}} d^{2}\left(u^{(n-1)}, C_{i}\right) \geq \eta_{R}\left(d\left(u^{(0)}, C\right)\right)
$$

for any $u^{(0)}$ with $\left\|u^{(0)}\right\| \leq R$ and $u^{(0)} \notin C$. The function $\eta_{R}$ is independent of the choice of the sequence $\beta^{(n)}$. 
Proof. Fix $r>0$ and consider the set

$$
B_{r, R}=\left\{u^{(0)} \in \mathbb{R}^{d}:\left\|u^{(0)}\right\| \leq R, d\left(u^{(0)}, C\right) \geq r\right\} .
$$

The proof will be completed if we show that for any $u^{(0)} \in B_{r, R}$ there exists a positive number $\eta_{R}(r)$ such that

$$
\max _{\substack{n \geq 1 \\ i \in I^{(n)}}} d^{2}\left(u^{(n-1)}, C_{i}\right) \geq \eta_{R}(r) .
$$

Suppose, by contradiction, that for any $m \in \mathbb{N}$ there exist vectors $u_{(m)}^{(0)} \in B_{R}$, and a sequence $\beta_{(m)}^{(n)} \in \mathcal{B}$, satisfying the assumptions of Lemma 1, such that

$$
\max _{\substack{n \geq 1 \\ i \in I_{(m)}^{(n)}}} d^{2}\left(u_{(m)}^{(n-1)}, C_{i}\right) \leq \frac{1}{m}
$$

where

$$
u_{(m)}^{(n)}=Q_{\beta_{(m)}^{(n)}}\left(u_{(m)}^{(n-1)}\right), \quad n \geq 1 .
$$

By compactness of $B_{r, R}$ we may assume that $x_{0}^{(m)} \stackrel{m}{\rightarrow} y$ and $y \in B_{r, R}$. Consider the sets $I_{(m)}^{(1)}$. Some indices of $\{1,2, \ldots, N\}$ occur for infinitely many $m$. Let $\mathcal{A}_{1}$ denote those indices. Clearly we may assume, eventually by restricting to large values of $m$, that only indices of $\mathcal{A}_{1}$ may occur in $I_{(m)}^{(1)}$, and each index does so infinitely many $m$. Therefore, fixing $n=1$ and taking the limit in (8) when $m \rightarrow \infty$ yield $y \in C_{i}$ for any $i \in \mathcal{A}_{1}$. If $\mathcal{A}_{1}=\{1,2, \ldots, N\}$, then $y \in C$, which is a contradiction with $y \in B_{r, R}$. Otherwise we have $\mathcal{A}_{1} \subsetneq\{1,2, \ldots, N\}$. Since for any fixed $m$ every index of $\{1,2, \ldots, N\}$ occurs in $I_{(m)}^{(n)}$ at least for one $n$, there exists the least number $l_{m}$ such that $I_{(m)}^{\left(l_{m}\right)} \backslash \mathcal{A}_{1} \neq \emptyset$. Observe that $Q_{\beta}(y)=y$ for any $\beta \in \mathcal{B}$ such that the set of active indices $I$ of $\beta$ is contained in $\mathcal{A}_{1}$. Therefore

$$
y=Q_{\beta_{(m)}^{(l m-1)}} Q_{\beta_{(m)}^{\left(l_{m}-2\right)}} \ldots Q_{\beta_{(m)}^{(2)}} Q_{\beta_{(m)}^{(1)}}(y) .
$$

Define

$$
\widetilde{u}_{(m)}^{(1)}:=u_{(m)}^{\left(l_{m}-1\right)}=Q_{\beta_{(m)}^{\left(l_{m}-1\right)}} Q_{\beta_{(m)}^{\left(l_{m}-2\right)}} \ldots Q_{\beta_{(m)}^{(2)}} Q_{\beta_{(m)}^{(1)}}\left(u_{(m)}^{(0)}\right) .
$$

Since the operators $Q_{\beta_{(m)}^{(k)}}$ are nonexpansive we obtain

$$
d\left(\widetilde{u}_{(m)}^{(1)}, y\right) \leq d\left(u_{(m)}^{(0)}, y\right)
$$

Hence $\widetilde{u}_{(m)}^{(1)} \stackrel{m}{\rightarrow} y$. 
Consider now the sets $I_{(m)}^{\left(l_{m}\right)}$. Each of these sets contains elements which do not belong to $\mathcal{A}_{1}$. Let $\mathcal{A}_{2}$ denote those indices outside $\mathcal{A}_{1}$ which occur for infinitely many values of $m$.. By restricting to large values of $m$ we may assume that only indices of $\mathcal{A}_{1}$ and $\mathcal{A}_{2}$ may occur in $I_{(m)}^{\left(l_{m}\right)}$ and all indices of $\mathcal{A}_{2}$ occur for infinitely many values of $m$. Set $n=l(m)+1$ in (8) $)$. Then since $\widetilde{u}_{(m)}^{(1)}=u_{(m)}^{\left(l_{m}-1\right)}$ we get

$$
\max _{i \in I_{(m)}^{\left(l_{m}\right)}} d^{2}\left(\widetilde{u}_{(m)}^{(1)}, C_{i}\right) \leq \frac{1}{m} .
$$

Therefore for any $i \in \mathcal{A}_{2}$ we have

$$
d^{2}\left(\widetilde{u}_{(m)}^{(1)}, C_{i}\right) \leq \frac{1}{m}
$$

for infinitely many $m$. As $\widetilde{u}_{(m)}^{(1)}$ tends to $y$, when $m \rightarrow \infty$, we obtain that $y \in C_{i}$ for any $i \in \mathcal{A}_{2}$. Therefore $y \in C_{i}$ for $i \in \mathcal{A}_{1} \cup \mathcal{A}_{2}$.

By repeating this argument at most $N$ times we get that $y \in C_{i}$ for any $i=1,2, \ldots, N$. Hence $y \in C$ which contradicts the fact that $y \in B_{r, R}$.

Let's return to the proof of Theorem 2. With no loss of generality we may assume that $0 \in C$. Fix $R>0$ and assume that $\left\|x^{(0)}\right\| \leq R$. Then since every operator $Q_{\alpha, \lambda}$ is nonexpansive we obtain $\left\|x^{(n)}\right\| \leq R$ for any $n$. Assume that

$$
\{1,2, \ldots, N\} \subset \bigcup_{j=n_{k-1}}^{n_{k}-1} I^{(j)} .
$$

Then combining Lemma 1 with $u^{(0)}=x^{\left(n_{k-1}\right)}$ and formula (7) yields

$$
\begin{array}{r}
\left\|x^{\left(n_{k}\right)}\right\|^{2} \leq\left\|x^{\left(n_{k-1}\right)}\right\|^{2}-\min _{\substack{n_{k-1}<n \leq n_{k} \\
i \in I^{(n)}}} \nu_{i}^{(n)} \max _{\substack{n_{k-1}<n \leq n_{k} \\
i \in I^{(n)}}} d^{2}\left(x^{(n-1)}, C_{i}\right) \\
\leq\left\|x^{\left(n_{k-1}\right)}\right\|^{2}-\nu^{(k)} \eta_{R}\left(d\left(x^{\left(n_{k-1}\right)}, C\right)\right)
\end{array}
$$

This implies that the series

$$
\sum_{k=1}^{\infty} \nu^{(k)} \eta_{R}\left(d\left(x^{\left(n_{k-1}\right)}, C\right)\right)
$$

is convergent. Since the operators $Q_{\beta}$ are nonexpansive, the sequence $d\left(x^{(n)}, C\right)$ is nonincreasing. Therefore, by assumptions made on the coefficients $\nu_{i}^{(n)}$, we obtain that $\eta_{R}\left(d\left(x^{(n)}, C\right)\right) \stackrel{n}{\rightarrow} 0$. Hence $d\left(x^{(n)}, C\right) \stackrel{n}{\rightarrow} 0$. 
Since $x^{(n)}$ is bounded, it contains a convergent subsequence $x^{\left(n_{m}\right)}$. Denote its limit by $c$. Then $c \in C$. By Proposition 1 the sequence $\left\|x^{(n)}-c\right\|$ is nonincreasing. Therefore, it tends to zero, i.e. $x^{(n)} \stackrel{n}{\rightarrow} c$.

\section{Perturbation}

Proposition 2. Let a sequence $\widetilde{\beta}^{(n)} \in \mathcal{B}$ be given. Assume that for any $m \in \mathbb{N}$ and $x \in \mathbb{R}^{d}$ the sequence

$$
Q_{\widetilde{\beta}^{(n)}} Q_{\widetilde{\beta}(n-1)} \ldots Q_{\widetilde{\beta}(m)}(x)
$$

is convergent to an element of $C$ as $n \rightarrow \infty$. Let sequences $\beta^{(n)} \in \mathcal{B}$ satisfy

$$
\sum_{n=1}^{\infty} \sum_{i=1}^{N}\left|\beta_{i}^{(n)}-\widetilde{\beta}_{i}^{(n)}\right|<\infty .
$$

Then for any $x \in \mathbb{R}^{d}$ the sequence

$$
Q_{\beta^{(n)}} Q_{\beta^{(n-1)}} \ldots Q_{\beta^{(1)}}(x)
$$

is convergent to an element of $C$ as $n \rightarrow \infty$.

Proof. By (1) we have

$$
\begin{aligned}
d\left(Q_{\beta^{(k)}}(y), Q_{\widetilde{\beta}^{(k)}}(y)\right) \leq \sum_{j=1}^{N}\left|\beta_{j}^{(k)}-\widetilde{\beta}_{j}^{(k)}\right| \mid\left\|P_{C_{j}} y-y\right\| & \\
& \leq \sum_{j=1}^{N}\left|\beta_{j}^{(k)}-\widetilde{\beta}_{j}^{(k)}\right| d(y, C) .
\end{aligned}
$$

Denote for simplicity

$$
Q_{n}:=Q_{\beta^{(n)}}, \quad \widetilde{Q}_{n}:=Q_{\widetilde{\beta}^{(n)}}
$$

and

$$
x^{(m)}=Q_{m} Q_{m-1} \ldots Q_{1}(x) .
$$


Then

$$
\begin{aligned}
& d\left(x^{(n)}, C\right)=d\left(Q_{n} Q_{n-1} \ldots Q_{m+1}\left(x^{(m)}\right), C\right) \\
& \leq \sum_{k=m+1}^{n} d\left(\widetilde{Q}_{n} \ldots \widetilde{Q}_{k+1} \widetilde{Q}_{k}\left(x^{(k-1)}\right), \widetilde{Q}_{n} \ldots \widetilde{Q}_{k+1} Q_{k}\left(x^{(k-1)}\right)\right) \\
& \quad+d\left(\widetilde{Q}_{n} \widetilde{Q}_{n-1} \ldots \widetilde{Q}_{m+1}\left(x^{(m)}\right), C\right) \\
& \leq \sum_{k=m+1}^{n} d\left(\widetilde{Q}_{k}\left(x^{(k-1)}\right), Q_{k}\left(x^{(k-1)}\right)\right)+d\left(\widetilde{Q}_{n} \widetilde{Q}_{n-1} \ldots \widetilde{Q}_{m+1}\left(x^{(m)}\right), C\right) \\
& \leq \sum_{k=m+1}^{n} \sum_{j=1}^{N}\left|\beta_{j}^{(k)}-\widetilde{\beta}_{j}^{(k)}\right| d\left(\widetilde{x}^{(k-1)}, C\right)+d\left(Q_{n} Q_{n-1} \ldots Q_{m+1}\left(\widetilde{x}^{(m)}\right), C\right) \\
& \leq \sum_{k=m+1}^{n} \sum_{j=1}^{N}\left|\beta_{j}^{(k)}-\widetilde{\beta}_{j}^{(k)}\right| d(x, C)+d\left(\widetilde{Q}_{n} \widetilde{Q}_{n-1} \ldots \widetilde{Q}_{m+1}\left(x^{(m)}\right), C\right)
\end{aligned}
$$

Now the conclusion follows from the assumptions. Indeed, we may assume that $d(x, C)>0$ as otherwise $x^{(n)}=x \in C$ for any $n$. Let $m$ be large so that

$$
\sum_{k=m+1}^{\infty} \sum_{j=1}^{N}\left|\beta_{j}^{(k)}-\widetilde{\beta}_{j}^{(k)}\right|<\frac{\varepsilon}{2 d(x, C)} .
$$

Next let $n$ be large so that

$$
d\left(\widetilde{Q}_{n} \widetilde{Q}_{n-1} \ldots \widetilde{Q}_{m+1}\left(x^{(m)}\right), C\right)<\varepsilon / 2 .
$$

Thus $d\left(x^{(n)}, C\right)<\varepsilon$ for $n$ large. Hence $d\left(x^{(n)}, C\right) \rightarrow 0$ as $n \rightarrow \infty$. This implies that $x^{(n)}$ tends to a point in $C$ (see the end of the proof of Theorem 2).

Corollary 1. Fix a family of convex sets $C_{1}, \ldots, C_{N}$ with nonempty intersection $C$. Given a sequence $\beta^{(n)} \in \mathcal{B}$. Let $I^{(n)}$ denote the set of active indices for $\beta^{(n)}$ and let $J^{(n)}$ be a sequence of subsets of $I^{(n)}$ such that

$$
\sum_{n=1}^{\infty} \sum_{i \in I^{(n)} \backslash J^{(n)}} \beta_{i}^{(n)}<+\infty .
$$

Assume that every index $i \in\{1,2, \ldots, N\}$ occurs in $J^{(n)}$ for infinitely many $n$. Let $n_{k}$ be positive integers such that $n_{k-1}<n_{k}$ and

$$
\{1,2, \ldots, N\} \subset J^{\left(n_{k-1}\right)} \cup J^{\left(n_{k-1}+1\right)} \cup \ldots \cup J^{\left(n_{k}-1\right)},
$$


i.e. every index occurs at least once for $n$ such that $n_{k-1} \leq n<n_{k}$. For

$$
\nu_{i}^{(n)}=\frac{2 \beta_{i}^{(n)}\left(2-\sum_{k=1}^{N} \beta_{k}^{(n)}\right)}{\beta_{i}^{(n)}+2-\sum_{k=1}^{N} \beta_{k}^{(n)}}
$$

let

$$
\nu_{J}^{(k)}=\min \left\{\nu_{i}^{(n)} \mid n_{k-1}<n \leq n_{k}, i \in J^{(n)}\right\} .
$$

Assume that

$$
\sum_{k=1}^{\infty} \nu_{J}^{(k)}=+\infty
$$

Then for any $x^{(0)} \in \mathbb{R}^{d}$ the sequence $x^{(n)}$ defined as

$$
x^{(n)}=Q_{\beta^{(n)}}\left(x^{(n-1)}\right), \quad n \geq 1
$$

is convergent to a point in $C$.

Proof. Define

$$
\widetilde{\beta}_{i}^{(n)}= \begin{cases}\beta_{i}^{(n)} & \text { if } i \in J^{(n)} \\ 0 & \text { if } i \in I^{(n)} \backslash J^{(n)}\end{cases}
$$

Clearly we have

$$
\sum_{k=1}^{N} \widetilde{\beta}_{k}^{(n)} \leq \sum_{k=1}^{N} \beta_{k}^{(n)}
$$

Hence $\widetilde{\nu}_{i}^{(n)} \geq \nu_{i}^{(n)}$ for $i \in J^{(n)}$, which implies $\widetilde{\nu}^{(k)} \geq \nu_{J}^{(k)}$. Therefore, the sequence $\widetilde{\beta}^{(n)}$ satisfies the assumptions of Theorem 2 . Therefore the sequence

$$
Q_{\widetilde{\beta}^{(n)}} Q_{\widetilde{\beta}^{(n-1)}} \ldots Q_{\widetilde{\beta}^{(m)}}(x)
$$

is convergent to an element of $C$ as $n \rightarrow \infty$. The sequences $\beta^{(n)}$ and $\widetilde{\beta}^{(n)}$ satisfy

$$
\sum_{n=1}^{\infty} \sum_{i=1}^{N}\left|\widetilde{\beta}_{i}^{(n)}-\beta_{i}^{(n)}\right|<\infty .
$$

Therefore applying Proposition 1 concludes the proof.

Example. Consider $N=3$ and

$$
\begin{aligned}
& \beta_{1}^{(2 n)}=\frac{1}{n^{2}}, \beta_{2}^{(2 n)}=\frac{1}{n}, \quad \beta_{3}^{(2 n)}=2-\frac{2}{n}, \\
& \beta_{1}^{(2 n+1)}=\frac{1}{n}, \quad \beta_{2}^{(2 n+1)}=\frac{1}{n^{2}}, \quad \beta_{3}^{(2 n+1)}=2-\frac{2}{n} .
\end{aligned}
$$


The scheme is 1 -intermittent, i.e. $I^{(n)}=\{1,2,3\}$ for any $n$. Observe that the assumptions of Theorem 2 are not satisfied. Now, consider this scheme as 2-intermittent and let

$$
J^{(2 n)}=\{2,3\} \quad J^{(2 n+1)}=\{1,3\} .
$$

Then we can apply Corollary 1 to obtain that this scheme leads to the convergence of the algorithm.

\section{INTERMitTENT CONTROL}

The assumptions of Theorem 2 depend on the behaviour of the coefficients $\beta_{i}^{(n)}$ where $i \in I^{(n)}$, i.e. those coefficients which are positive. Roughly the conclusion holds if these coefficients are not to small and the sums $s^{(n)}=\sum_{i=1}^{N} \beta_{i}^{(n)}$ do not approach the value 2 too fast. By Corollary 1 we can allow some small coefficients $\beta_{i}^{(n)}$ by using perturbation technique. However in special case of intermittent control and when the sums $s^{(n)}$ stay away from 2 we can entirely liberate ourselves from assumptions on all positive coefficients $\beta_{i}^{(n)}$.

Theorem 3. Fix a family of convex sets $C_{1}, \ldots, C_{N}$ with nonempty intersection $C$. Given a sequence $\beta^{(n)} \in \mathcal{B}$ such that

$$
s^{(n)}=\sum_{i=1}^{N} \beta_{i}^{(n)} \leq 2-\varepsilon
$$

for some constant $\varepsilon>0$. Let $I^{(n)}$ denote the set of active indices for $\beta^{(n)}$ and let $J^{(n)}$ be a sequence of subsets of $I^{(n)}$. Assume that there is a positive integer $p$ such that for any $k$ we have

$$
\{1,2, \ldots, N\} \subset I^{((k-1) p)} \cup I^{(k-1) p+1)} \cup \ldots \cup I^{(k p-1)} .
$$

Let

$$
\nu_{J}^{(k)}=\min \left\{\beta_{i}^{(n)} \mid(k-1) p<n \leq k p, i \in J^{(n)}\right\} .
$$

Assume that

$$
\sum_{k=1}^{\infty} \nu_{J}^{(k)}=+\infty
$$

Then for any $x^{(0)} \in \mathbb{R}^{d}$ the sequence $x^{(n)}$ defined as

$$
x^{(n)}=Q_{\beta^{(n)}}\left(x^{(n-1)}\right), \quad n \geq 1
$$

is convergent to a point in $C$.

Proof. First observe that since $s^{(n)} \leq 2-\varepsilon$ we have

$$
\frac{2 \varepsilon}{2+\varepsilon} \beta_{i}^{(n)} \leq \nu_{i}^{(n)}=\frac{2 \beta_{i}^{(n)}\left(2-s^{(n)}\right)}{\beta_{i}^{(n)}+2-s^{(n)}} \leq 2 \beta_{i}^{(n)} .
$$


Therefore we can replace the coefficients $\nu_{i}^{(n)}$ with $\beta_{i}^{(n)}$ when applying Theorem 2 and Corollary 1.

Let $n_{k}=k p$. If for

$$
\nu^{(k)}=\min \left\{\beta_{i}^{(n)} \mid(k-1) p<n \leq k p, i \in I^{(n)}\right\}
$$

we have

$$
\sum_{k=1}^{\infty} \nu^{(k)}=+\infty
$$

we can apply Theorem 2 to get the conclusion. Assume that

$$
\sum_{k=1}^{\infty} \nu^{(k)}<+\infty
$$

Let

$$
A=\left\{k \in \mathbb{N} \mid(\exists n)(\exists i)(k-1) p<n \leq k p, i \in I^{(n)} \backslash J^{(n)}, \nu^{(k)}=\beta_{i}^{(n)}\right\} .
$$

For every $k \in A$ choose $n_{k}$ and $i_{k}$ such that

$$
(k-1) p<n_{k} \leq k p, \quad i_{k} \in I^{(n)} \backslash J^{(n)}, \quad \nu^{(k)}=\beta_{i_{k}}^{\left(n_{k}\right)} .
$$

By (15) we have

$$
\sum_{k \in A}^{\infty} \beta_{i_{k}}^{\left(n_{k}\right)}<+\infty
$$

Define the new coefficients $\widetilde{\beta}_{i}^{(n)}$ by nullifying the coefficients $\beta_{i}^{(n)}$ for $i=i_{k}$ and $n=n_{k}$, i.e. let

$$
\widetilde{\beta}_{i}^{(n)}= \begin{cases}0 & \text { if } n=n_{k}, i=i_{k} \text { for some } k \\ \beta_{i}^{(n)} & \text { otherwise }\end{cases}
$$

By construction the sums $\widetilde{s}^{(k)}$ stay away from 2 since $\widetilde{s}^{(k)} \leq s^{(k)}$. Moreover $J^{(n)} \subset \widetilde{I}^{(n)}$. By Corollary 1 the convergence of the algorithm for the new coefficients implies the convergence for the original ones. Thus we can restrict ourselves to the coefficients $\widetilde{\beta}_{i}^{(n)}$. Clearly for $i \in J^{(n)}$ we have $\widetilde{\beta}_{i}^{(n)}=\beta_{i}^{(n)}$. If the new coefficients satisfy (14) we are done by Theorem 2. If not, we can perform the same transformation as before. By at most $p N$ iterations we will obtain a sequence to which we can apply Theorem 2 which differs from original sequence as in Corollary 1. 
Example Let $N=3$ and

$$
\begin{aligned}
& \beta_{1}^{(3 n)}=1, \quad \beta_{2}^{(3 n)}=\frac{1}{n}, \quad \beta_{2}^{(3 n)}=\frac{1}{n^{2}}, \\
& \beta_{1}^{(3 n+1)}=\frac{1}{n}, \quad \beta_{2}^{(3 n+1)}=1, \quad \beta_{2}^{(3 n+1)}=\frac{1}{n^{2}}, \\
& \beta_{1}^{(3 n+2)}=\frac{1}{n^{2}}, \quad \beta_{2}^{(3 n+2)}=\frac{1}{n}, \quad \beta_{2}^{(3 n+2)}=1 .
\end{aligned}
$$

We have $I^{(n)}=\{1,2,3\}$. Let

$$
J^{(3 n)}=\{1\}, \quad J^{(3 n+1)}=\{2\}, \quad J^{(3 n+2)}=\{1\}
$$

and $p=3$. We have $\nu_{J}^{(k)}=1$ for any $k$. Hence all the assumptions of Theorem 3 are satisfied.

Acknowledgment. I am very grateful to Andrzej Cegielski for his help, and in particular for turning my attention to the paper [1].

\section{REFERENCES}

[1] H. H. Bauschke, J. M. Borwein, On projection algorithms for solving convex feasibility problems, SIAM Rev. 38(3) (1996), 367-426.

Institute of Mathematics, University of Wroceaw, Pl. Grunwaldzki 2/4, 50-384 Wroceaw, Poland AND

Institute of Mathematics and Computer Science, University of Opole, ul. Oleska 48, 45-052 Opole, Poland

E-mail address: szwarc2@gmail.com 